\title{
Accelerated expansion of the Universe in Gauss-Bonnet gravity
}

\author{
M. H. Dehghan:* \\ Physics Department and Biruni Observatory, \\ Shiraz University, Shiraz 71454, Iran \\ and \\ Institute for Studies in Theoretical Physics and Mathematics (IPM) \\ P.O. Box 19395-5531, Tehran, Iran
}

\begin{abstract}
We show that in Gauss-Bonnet gravity with negative Gauss-Bonnet coefficient and without a cosmological constant, one can explain the acceleration of the expanding Universe. We first introduce a solution of the Gauss-Bonnet gravity with negative Gauss-Bonnet coefficient and no cosmological constant term in an empty $(n+1)$ dimensional bulk. This solution can generate a de Sitter spacetime with curvature $n(n+1) /\{(n-2)(n-3)|\alpha|\}$. We show that an $(n-1)$-dimensional brane embedded in this bulk can have an expanding feature with acceleration. We also considered a 4-dimensional brane world in a 5-dimensional empty space with zero cosmological constant and obtain the modified Friedmann equations. The solution of these modified equations in matter-dominated era presents an expanding Universe with negative deceleration and positive jerk which is consistent with the recent cosmological data. We also find that for this solution, the " $n$ " $t h$ derivative of the scale factor with respect to time can be expressed only in terms of Hubble and deceleration parameters.
\end{abstract}

\section{INTRODUCTION}

The idea of brane cosmology has attracted a great deal of attention recently. It may provide us a new solution for the so-called hierarchy problem or the source of dark energy and dark matter [1, 2]. This theory is also consistent with string theory, which suggests that matter and gauge interaction (described by an open string) may be localized on a brane,

*Electronic address: mhd@shirazu.ac.ir 
embedded into a higher dimensional spacetime, while the field represented by closed strings, in particular gravity, propagate in the whole of spacetime. In the brane world scenario, we live in a 4-dimensional (4-D) hypersurface embedded in a higher dimensional bulk spacetime.

Among many brane models, those proposed by Randall and Sundrum which are motivated by superstring/M-theory [3] are the most applicable ones. In their first model (RS I) [4], they proposed a mechanism to solve the hierarchy problem with two branes, while in their second model (RS II) [5], they consider a single brane with a positive tension, where 4-D Newtonian gravity is recovered at low energies even if the extra dimension is not compact.

Brane world cosmology underscores the need to consider gravity in higher dimensions. In this context one may use another consistent theory of gravity in any dimension with a more general action. This action may be written, for example, through the use of string theory. The effect of string theory on classical gravitational physics is usually investigated by means of a low energy effective action which describes gravity at the classical level [6]. This effective action consists of the Einstein-Hilbert action plus curvature-squared terms and higher powers as well, and in general gives rise to fourth order field equations and bring in ghosts. However, if the effective action contains the higher powers of curvature in particular combinations, then only second order field equations are produced and consequently no ghosts arise [7]. The effective action obtained by this argument is precisely of the form proposed by Lovelock [8]. The appearance of higher derivative gravitational terms can be seen also in the renormalization of quantum field theory in curved spacetime [9].

These facts provide a strong motivation for investigating how the usual features of brane world cosmology are modified by more general gravitational theories such as Lovelock gravity. In this context, many authors are extensively studying the brane world cosmology in GaussBonnet gravity [10]. In these analysis, one needs the solutions of Gauss-Bonnet gravity in the bulk spacetime. Because of the nonlinearity of the field equations, it is very difficult to find out nontrivial exact analytical solutions of Einstein's equation with higher curvature terms. However, a few exact solutions of this theory have been obtained [11, 12].

On the other hand, it seems established that at the present epoch the Universe expands with acceleration instead of deceleration along the scheme of standard Friedmann model. This follows directly from the observation of high red-shift supernova [13] and indirectly from the measurement of angular fluctuations of cosmic microwave background fluctuations 14]. The latter shows that the total mass/energy density of the Universe is very close to 
critical value one $(\Omega=1)$, while the observations of the Universe in large scale structure indicate that normal gravitating (visible and invisible) matter can contribute only $30 \%$ into the total one. Thus, one concludes that the remaining $70 \%$ is some mysterious agent that creates the cosmological acceleration. The simplest suggestion is that the source of this acceleration is the vacuum energy (cosmological constant). However, it meets the two well known cosmological and coincidence problems [15]. A second approach is the consideration of cosmological components as a dynamical term. This scheme, usually called quintessence, can be achieved by adding a scalar field into Einstein gravity. Several forms of potential achieve quintessence prescriptions but non of them seems to be directly related to some fundamental quantum field theory. A third approach is to obtain a comprehensive model derived from some effective theory of quantum gravity which through an inflationary period results into the today accelerated expansion of the Universe. From this point of view, gravitational theories including higher order curvature terms naturally come into the game 16]. However, some of these modified gravitational theory have their own problems [17].

Here we want to explain accelerating Universe by use of the Gauss-Bonnet gravity without a cosmological constant term or a scalar field or any other kind of dark energy. Thus, we restrict ourself to the second and third terms of Lovelock gravity. The second term is the Einstein-Hilbert term, while the third term is known as the Gauss-Bonnet term. From a geometric point of view, the combination of the Gauss-Bonnet terms constitutes, for fivedimensional spacetimes, the most general Lagrangian producing second order field equations, as in the four-dimensional gravity where the Einstein-Hilbert action is the most general Lagrangian producing second order field equations [18].

The outline of our paper is as follows. We give a brief review of the field equations in Sec. [II. In Sec. III] we introduce a solution of the Gauss-Bonnet gravity with negative GaussBonnet coefficient and no cosmological constant in an empty $(n+1)$-dimensional bulk, and show that this solution can generate a de Sitter spacetime. In Sec. IV we first introduce the modified Friedmann equations, and then obtain the solutions of these equation in matterdominated era, which can discuss today accelerated expansion of the Universe. We finish our paper with some concluding remarks. 


\section{FIELD EQUATIONS IN GAUSS-BONNET GRAVITY}

The most fundamental assumption in standard general relativity is the requirement that the field equations be generally covariant and contain at most second order derivative of the metric. Based on this principle, the most general Lagrangian in arbitrary dimensions is the Lovelock Lagrangian. The Lagrangian of the Lovelock theory, which is the sum of dimensionally extended Euler densities, may be written as

$$
\mathcal{L}_{G}=\frac{1}{2} \sum_{i=1}^{[n / 2]} c_{i} \mathcal{L}_{i}
$$

where $c_{i}$ is an arbitrary constant, $[x]$ denotes the integer part of $x$, and $\mathcal{L}_{i}$ is the Euler density of a $2 i$-dimensional manifold,

$$
\mathcal{L}_{i}=(-2)^{-i} \delta_{c_{1} d_{1} \ldots c_{i} d_{i}}^{a_{1} b_{1} \ldots a_{i} b_{i}} \mathcal{R}_{a_{1} b_{1}}^{c_{1} d_{1}} \ldots . \mathcal{R}_{a_{i} b_{i}}{ }^{c_{i} d_{i}}
$$

In Eq. (2) $\mathcal{R}_{a b c d}$ is the Riemann tensor and $\delta_{c_{1} d_{1} \ldots c_{i} d_{i}}^{a_{1} b_{1} \ldots a_{i} b_{i}}$ is the totally antisymmetric product of $i$ Kronecker deltas, normalized to values 0 and \pm 1 . Here the first term $c_{1} \mathcal{L}_{1}=\mathcal{R}$, is just the Einstein-Hilbert, and the second term $c_{2} \mathcal{L}_{2}=\alpha\left(\mathcal{R}_{\mu \nu \gamma \delta} \mathcal{R}^{\mu \nu \gamma \delta}-4 \mathcal{R}_{\mu \nu} R^{\mu \nu}+\mathcal{R}^{2}\right)$ gives us the Gauss-Bonnet term. Of course, one may add a constant term to the above Lagrangian, playing the role of cosmological constant term. But, as we mentioned before this creates its own problems and therefore we don't disturb ourself with it. The Lagrangian of Gauss-Bonnet gravity with zero cosmological constant is

$$
\mathcal{L}_{G}=\frac{1}{2}\left\{\mathcal{R}+\alpha\left(\mathcal{R}_{\mu \nu \gamma \delta} \mathcal{R}^{\mu \nu \gamma \delta}-4 \mathcal{R}_{\mu \nu} \mathcal{R}^{\mu \nu}+\mathcal{R}^{2}\right)\right\}
$$

where $\mathcal{R}, \mathcal{R}_{\mu \nu \rho \sigma}$, and $\mathcal{R}_{\mu \nu}$ are the Ricci scalar and Riemann and Ricci tensors of the spacetime, and $\alpha$ is the Gauss-Bonnet coefficient with dimension (length) ${ }^{2}$. Here we restrict ourselves to the case $\alpha<0$.

Let us suppose, as would be in the co-dimension one brane world scenario, that the $(n+1)$-dimensional bulk, $\mathcal{M}$ is split into two parts by a hypersurface $\Sigma$, whose two sides will be denoted by $\Sigma_{ \pm}$. Their normal unit vector, $n^{\mu}$, will be taken to point away from the surface and into the adjacent space. The gravitational action for the spacetime $\left(\mathcal{M}, g_{\mu \nu}\right)$ with boundary $\left(\Sigma, h_{a b}\right)$ can be written as 19,20$]$

$$
I_{G}=\frac{1}{2 \kappa^{2}} \int_{\mathcal{M}} d x^{n+1} \sqrt{-g} \mathcal{L}_{G}-\frac{1}{\kappa^{2}} \int_{\Sigma_{ \pm}} d x^{n} \sqrt{-h}\left[K+2 \alpha\left(J-2 G^{a b} K_{a b}\right)\right],
$$


where $K_{\mu \nu}$ is the extrinsic curvature of the hypersurface $\Sigma$ with induced metric $h_{a b}, G^{a b}$ is the Einstein tensor of the metric $h_{a b}$ and $J$ is the trace of

$$
J_{a b}=\frac{1}{3}\left(2 K K_{a c} K_{b}^{c}+K_{c d} K^{c d} K_{a b}-2 K_{a c} K^{c d} K_{d b}-K^{2} K_{a b}\right) .
$$

The first term in Eq. (33) is the Einstein-Gauss-Bonnet terms, and the second term is the boundary term which is chosen such that the variational principle is well-defined [19]. This term is, in fact, the generalization of Gibbons-Hawking boundary term in Einstein gravity. If we also include a matter contribution to the action

$$
I_{\text {mat }}=-\int_{\mathcal{M}} d x^{n+1} \sqrt{-g} \mathcal{L}_{m}^{(\mathcal{M})}-\int_{\Sigma} d x^{n} \sqrt{-h} \mathcal{L}_{m}^{(\Sigma)},
$$

then the variation of the total action $I=I_{G}+I_{\text {mat }}$ over the metric tensor $g_{\mu \nu}$ gives the equation of gravitational fields in the bulk as

$$
\begin{aligned}
& \mathcal{R}_{\mu \nu}-\frac{1}{2} g_{\mu \nu} \mathcal{R}-\alpha\left\{\frac{1}{2} g_{\mu \nu}\left(\mathcal{R}_{\kappa \lambda \rho \sigma} \mathcal{R}^{\kappa \lambda \rho \sigma}-4 \mathcal{R}_{\rho \sigma} \mathcal{R}^{\rho \sigma}+\mathcal{R}^{2}\right)\right. \\
& \left.-2 \mathcal{R} \mathcal{R}_{\mu \nu}+4 \mathcal{R}_{\mu \lambda} \mathcal{R}^{\lambda}{ }_{\nu}+4 \mathcal{R}^{\rho \sigma} \mathcal{R}_{\mu \rho \nu \sigma}-2 \mathcal{R}_{\mu}{ }^{\rho \sigma \lambda} \mathcal{R}_{\nu \rho \sigma \lambda}\right\}=\kappa^{2} \mathcal{T}_{\mu \nu}
\end{aligned}
$$

where $\mathcal{T}_{\mu \nu}$ is the energy momentum of the bulk defined by $\mathcal{T}_{\mu \nu}=2 \delta \mathcal{L}_{m}^{(\mathcal{M})} / \delta g^{\mu \nu}-g_{\mu \nu} \mathcal{L}_{m}^{(\mathcal{M})}$. Assuming the $Z_{2}$-symmetry for the brane, then the variation of total action with respect to $h_{a b}$ gives [20]

$$
K_{a b}-K h_{a b}+2 \alpha\left(3 J_{a b}-J h_{a b}+2 P_{a c b d} K^{c d}\right)=-\frac{\kappa^{2}}{2} T_{a b},
$$

where $T_{a b}=2 \delta \mathcal{L}_{m}^{(\Sigma)} / \delta g^{a b}-g_{a b} \mathcal{L}_{m}^{(\Sigma)}$ is the energy momentum in the hypersurface and $P_{a c b d}$ is the divergence free part of the Riemann tensor of the metric $h_{a b}$

$$
P_{a c b d}=R_{a c b d}+2 h_{a[c} R_{d] b}+2 h_{b[d} R_{c] a}+R h_{a[c} h_{d] b} .
$$

Also it is worthwhile to mention that the energy-momentum conservation on the hypersurface may be written as

$$
D^{b} T_{a b}=-2 \mathcal{T}_{\mu \nu} h_{a}{ }^{\mu} n^{\nu}
$$

where $D^{b}$ denotes the covariant derivative corresponding to $h_{a b}$.

\section{THE DE SITTER SOLUTIONS IN AN EMPTY BULK}

Here we want to obtain the $(n+1)$-dimensional solutions of Eq. (7) in vacuum, which generates a de sitter spacetime. We assume that the $(n+1)$-dimensional spacetime be 
isotropic and homogenous, i.e. it has a maximally symmetric $n$-dimensional space. Since we are interested in cosmological solutions, we take a metric of the form

$$
d s^{2}=-d t^{2}+f^{2}(t)\left[\frac{d r^{2}}{1-k r^{2}}+r^{2} d \Omega^{2}\right]
$$

where $d \Omega^{2}$ is the metric of an $(n-1)$-sphere and $k$ is a constant which can take only the value 1,0 and -1 . To find the function $F(t)$, one may use any components of Eq. (7). The simplest equation is the $t$-component of these equations which can be written as

$$
\left(\dot{f}^{2}+k\right)\left[|\alpha|(n-2)(n-3)\left(\dot{f}^{2}+k\right)-f^{2}\right]=0
$$

where the overdot denotes a derivative with respect to the $t$ coordinate. The solutions of Eq. (12) can be written as

$$
f(t)=\frac{1}{2 \sqrt{A B}}\left\{A \exp \left(\frac{t}{\sqrt{(n-2)(n-3)|\alpha|}}\right)+B k|\alpha| \exp \left(-\frac{t}{\sqrt{(n-2)(n-3)|\alpha|}}\right)\right\},
$$

where $A$ and $B$ are two arbitrary functions. Also for negative $k, f(t)=\sqrt{-k} t$ is another solution. The function $f(t)$ given in Eq. (13) shows that the $(n+1)$-dimensional bulk spacetime has an expanding feature. For example, if one choose a brane in the hypersurface of $r=r_{0}$, then the volume of $(n-1)$-brane is

$$
V_{n-1}=\frac{2 \pi^{n / 2}}{\Gamma(n / 2)} f^{n-1}(t)
$$

It is worthwhile to note that the function $f(t)$ has exponential behavior for the case of flat space with $k=0$. That is

$$
f(t)=A \exp \left(\frac{t}{\sqrt{(n-2)(n-3)|\alpha|}}\right) .
$$

Also, one may note that the spacetime is asymptotically de Sitter for an arbitrary value of $k$. In these two cases $(k=0$ and $t \rightarrow \infty)$ the curvature of the spacetime is $n(n+1) /\{(n-$ 2) $(n-3)|\alpha|\}$, which means that the spacetime is de Sitter.

\section{MODIFIED FRIEDMANN EQUATIONS IN GAUSS-BONNET GRAVITY}

Now we consider a 5-D bulk spacetime with a single 4-D hypersurface at $r=r_{0}$. The most general gravitational equation in five dimension which is symmetric, divergence free 
and linear in second derivative of the metric is Einstein-Gauss-Bonnet equations. Thus, we use these equations in order to consider the 4-D brane world cosmology. The brane world $\left(\mathcal{B}, h_{\mu \nu}\right)$ is located at the hypersurface $r=r_{0}$, and its induced metric is $h_{\mu \nu}=g_{\mu \nu}-n_{\mu} n_{\nu}$, where $n^{\mu}=\left(0, \sqrt{g_{r r}}, 0,0,0\right)$ is the normal unit vector to the brane. Since we assume to have a perfect fluid in the brane world at $r=r_{0}$, the bulk has a spherical symmetric 4-D space. Thus, the bulk metric can be written as:

$$
d s^{2}=-d t^{2}+f^{2}(t)\left[\frac{d r^{2}}{c^{2}(r)}+d \chi^{2}+b^{2}(\chi)\left(d \phi^{2}+\sin ^{2} \phi d \psi^{2}\right)\right],
$$

where $b(\chi)$ for the closed, flat and open Universe is

$$
b(\chi)= \begin{cases}\sin \chi & k=+1 \\ \chi & k=0 \\ \sinh \chi & k=-1\end{cases}
$$

respectively. The induce metric is

$$
d s^{2}=-d t^{2}+a^{2}(t)\left[d \chi^{2}+b^{2}(\chi)\left(d \phi^{2}+\sin ^{2} \phi d \psi^{2}\right)\right],
$$

where $a(t)=r_{0} f(t)$. The nonvanishing components of the extrinsic curvature of the hypersurface are

$$
\begin{aligned}
K_{\chi \chi} & =c_{0} a(t), \\
K_{\phi \phi} & =c_{0} a(t) b^{2}(\chi), \\
K_{\psi \psi} & =c_{0} a(t) b^{2}(\chi) \sin ^{2} \phi,
\end{aligned}
$$

where $c_{0}=c\left(r_{0}\right)$. Using Eqs. (8) and (19), and the expression of the energy-momentum tensor of a perfect fluid

$$
T_{a b}=(\rho+p) u_{a} u_{b}+p h_{a b},
$$

one obtains the following modified Friedmann equations:

$$
\begin{aligned}
& \frac{\ddot{a}}{a}=\frac{4 c_{0}-\kappa^{2} p a}{16 c_{0}|\alpha|}, \\
& 24 c_{0}|\alpha|\left(k+\dot{a}^{2}\right)+16 c_{0}^{3}|\alpha|-6 c_{0} a^{2}-\kappa^{2} \rho a^{3}=0 .
\end{aligned}
$$

Equation (21) indicates that in matter-dominated era $(p=0)$, our Universe expands with acceleration instead of deceleration along the scheme of standard Friedmann model. To be 
more clear we obtain a class of solutions of Eqs. (21) and (22). First, we go through the conservation of energy-momentum. Using Eqs. (10) and (20) for the metric (16), and the fact that the bulk is empty, one obtains

$$
d\left(\rho a^{3}\right)=-p d a^{3}
$$

Given an equation of state $p=p(\rho)$, one can use Eq. (23) to determine $\rho$ as a function of $a$. Knowing $\rho$ as a function of $a$, one can find $a(t)$ for all time by solving Eq. (22). Incidentally, the solution $a(t)$ determined in this way will automatically satisfy Eq. (21), for by differentiating Eq. (22) with respect to time and using Eq. (23), one obtains Eq. (21).

\section{A. Matter dominated era}

Assuming the energy density of Universe is dominated by non-relativistic matter with negligible pressure, then $\rho \propto a^{-3}$, and therefore Eq.(22) can be written as

$$
24 c_{0}|\alpha|\left(k+\dot{a}^{2}\right)-6 c_{0} a^{2}+16 c_{0}^{3}|\alpha|-\kappa^{2} \rho_{0} a_{0}^{3}=0,
$$

where $\rho_{0}$ and $a_{0}$ are the values of $\rho$ and $a$ at the present. Equation (24) can be solved exactly. One can write the solutions of this equation as

$$
a(t)=A \exp \left(\frac{t}{2 \sqrt{|\alpha|}}\right)+B_{k} \exp \left(-\frac{t}{2 \sqrt{|\alpha|}}\right) .
$$

where $B_{k}=\left(16 c_{0}^{3}|\alpha|+24|\alpha| k c_{0}-\kappa^{2} \rho_{0} a_{0}^{3}\right) /(24 A)$. Since $c_{0}$ is arbitrary, therefore $A$ and $B_{k}$ are two arbitrary constants. The arbitrary constants $A$ and $B_{k}$, and the Gauss-Bonnet parameter $\alpha$ may be fixed by observational data. In order to give the way of fixing $A, B_{k}$ and $\alpha$, it is more convenient to work with the Hubble $\left(H \equiv a^{-1} \dot{a}\right)$, deceleration $\left(q \equiv-H^{-2} a^{-1} \ddot{a}\right)$, jerk $\left(j \equiv H^{-3} a^{-1} a \dddot{a}\right), \operatorname{snap}\left(s \equiv H^{-4} a^{-1} \dddot{a}\right)$ and $\ldots$ parameters [21]. It is remarkable to note that for $a(t)$ given in Eq. (25), one encounters only with Hubble and deceleration parameters $\left(H_{0}\right.$ and $\left.q_{0}\right)$ at present epoch. Indeed, for $a(t)$ given in Eq. (25) $d^{n} a / d t^{n}=H^{n} q^{[n / 2]}$, and therefore the Taylor expansion of $a(t)$ is

$$
a(t)=a_{0} \sum_{n=0}^{\infty} \frac{1}{n !} H_{0}{ }^{n}\left|q_{0}\right|^{[n / 2]}\left(t-t_{0}\right)^{n},
$$

where $[n / 2]$ denotes the integer part of $n / 2$. 
In order to use the astronomical data for fixing $\left(A, B_{k}, \alpha\right)$ or equivalently $\left(a_{0}, H_{0}, q_{0}\right)$, one may use the relation between the luminosity distance $d_{L}$ and the redshift $z$ of a luminous source. It is easy to show that (see the Appendix):

$$
\begin{aligned}
d_{L}=\frac{z}{H_{0}}\{1 & +\frac{1}{2}\left(1-q_{0}\right) z+\frac{1}{6}\left[1-2 q_{0}-3 q_{0}^{2}+\frac{k}{H_{0}^{2} a_{0}^{2}}\right] z^{2} \\
& \left.+\frac{1}{24}\left[1-7 q_{0}-24 q_{0}^{2}-15 q_{0}^{3}+\frac{2 k\left(1+q_{0}\right)}{H_{0}^{2} a_{0}^{2}}\right] z^{3}+\ldots\right\}
\end{aligned}
$$

Having enough astronomical data, one can determines $\left(a_{0}, H_{0}, q_{0}\right)$ and therefore $\left(A, B_{k}\right.$, $\alpha$ ). The solution (25) indicates that during the matter-dominated era, the Universe expands with acceleration instead of deceleration with positive jerk. This feature is consistent with the observation of high red-shift supernova or the measurement of angular fluctuations of cosmic microwave background fluctuations [13, 14, 22]. Also, one may note that for $a(t)$ given in Eq. (25) $H_{\infty}=(2 \sqrt{|\alpha|})^{-1}$ and $s_{\infty}=j_{\infty}=-q_{\infty}=1$ as $t \rightarrow \infty$.

\section{CLOSING REMARKS}

In this paper, we added the Gauss-Bonnet term with negative coefficient to the Einstein action without a cosmological constant term, and introduced a solution of the field equations in an empty $(n+1)$-dimensional bulk. We found that this solution is de Sitter with constant curvature $n(n+1) / 2|\alpha|$ for the case of flat space $(k=0)$ or as $t \rightarrow \infty$. We showed that an $(n-1)$-dimensional brane embedded in the bulk can have an expanding feature with acceleration instead of deceleration.

We also considered the 4-dimensional brane world in a 5-dimensional empty space with zero cosmological constant. This was done in Gauss-Bonnet gravity which has the most general gravitational field equation in five dimension. We obtained the modified Friedmann equations and showed that these two equations are not functionally independent if one use the conservation of energy. Equation (21) indicates that our Universe expands with acceleration instead of deceleration in matter-dominated era. This was done without need to any mysterious fluid with large negative pressure or the cosmological constant. We also obtained the solutions of the modified Friedmann equations for the matter-dominated era. This solution presents an expanding Universe with positive acceleration and jerk which is consistent with the recent cosmological data. We also found that $a(t)$ can be expressed in 
terms of $a_{0}, H_{0}$ and $q_{0}$ only, and all the other parameters such as jerk, snap and so on can be written in terms of $H_{0}$ and $q_{0}$.

As stated before, the Gauss-Bonnet gravity is the most general gravitational field equation in five dimension. In higher dimension one should use more terms for action in Lovelock theory. The application of the above method in higher dimension, which needs the use of Lovelock gravity with more gravitational terms remains to be carried out in future.

\section{APPENDIX}

In this appendix, we obtain the relation between the luminosity distance $d_{L}$ and the redshift $z$. This relation has been derived for Robertson-Walker spacetime in [21]. Here we obtain it for the metric (18). The physical distance travelled by a photon emitted at time $t_{*}$ and absorbed at the current epoch $t_{0}$ in term of $z$ is [21]

$$
\begin{aligned}
D= & \frac{z}{H_{0}}\left\{1-\left[1+\frac{q_{0}}{2}\right] z+\left[1+q_{0}+\frac{q_{0}^{2}}{2}-\frac{j_{0}}{6}\right] z^{2}\right. \\
& \left.-\left[1+\frac{3}{2} q_{0}\left(1+q_{0}\right)+\frac{5}{8} q_{0}^{3}-\frac{1}{2} j_{0}-\frac{5}{12} q_{0} j_{0}-\frac{s_{0}}{24}\right] z^{3}+O\left(z^{4}\right)\right\},
\end{aligned}
$$

while $d_{L}$ can be written as

$$
d_{L}=a\left(t_{0}\right)^{2} \frac{r_{0}}{a\left(t_{*}\right)}=\frac{a_{0}^{2}}{a\left(t_{0}-D\right)} b(\chi)=\frac{a_{0}^{2}}{a\left(t_{0}-D\right)}\left\{1-\frac{1}{6} \chi^{3}+O\left(\chi^{5}\right)\right\} .
$$

Recall that for a null geodesic in the spacetime (18)

$$
\begin{gathered}
\chi_{0}=\int_{t_{*}}^{t_{0}} \frac{d t}{a(t)}=\int_{t_{0}-D}^{t_{0}} \frac{c d t}{a_{0}}\left\{1+H_{0}\left(t_{0}-t\right)+\left[\frac{2+q_{0}}{2} H_{0}^{2}\right]\left(t_{0}-t\right)^{2}\right. \\
\left.+\left[\frac{6\left(1+q_{0}\right)+j_{0}}{6} H_{0}^{3}\right]\left(t_{0}-t\right)^{3}+O\left[\left(t_{0}-t\right)^{4}\right]\right\} \\
=\frac{D}{a_{0}}\left\{1+\frac{1}{2} H_{0} D+\left[\frac{2+q_{0}}{6}\right]\left(H_{0} D\right)^{2}\right. \\
\left.+\left[\frac{6\left(1+q_{0}\right)+j_{0}}{24}\right]\left(H_{0} D\right)^{3}+O\left[\left(H_{0} D\right)^{4}\right]\right\} .
\end{gathered}
$$

Now using Eqs. (28)-(30), one obtains

$$
\begin{aligned}
d_{L}(z)= & \frac{z}{H_{0}}\left\{1+\frac{1}{2}\left[1-q_{0}\right] z-\frac{1}{6}\left[1-q_{0}-3 q_{0}^{2}+j_{0}+\frac{k c^{2}}{H_{0}^{2} a_{0}^{2}}\right] z^{2}\right. \\
& +\frac{1}{24}\left[2-2 q_{0}-15 q_{0}^{2}-15 q_{0}^{3}+5 j_{0}+10 q_{0} j_{0}+s_{0}+\frac{2 k c^{2}\left(1+3 q_{0}\right)}{H_{0}^{2} a_{0}^{2}}\right] z^{3} \\
& \left.+O\left(z^{4}\right)\right\} .
\end{aligned}
$$


[1] A. Lukas, B. A. Ovrut, K. S. Stelle, and D. Waldram, Phys. Rev. D 59, 086001 (1999); A. Lukas, B. A. Ovrut, and D. Waldram, Phys. Rev. D 60, 086001 (2000).

[2] P. Binetruy, C. Deffayet, and D. Langlois, Nucl. Phys. B565, 269 (2000).

[3] P. Horava and E. Witten, Nucl. Phys B475, 94 (1996).

[4] L. Randall and R. Sundrum, Phys. Rev. Lett. 83, 3370 (1999).

[5] L. Randall and R. Sundrum, Phys. Rev. Lett. 83, 4690 (1999).

[6] P. Candelas, G. T. Horowitz, A. Strominger, and E. Witten, Nucl. Phys. B258, 46 (1985); M. B. Greens, J. H. Schwarz and E. Witten, Superstring Theory (Cambridge University Press, Cambridge, England, 1987); D. Lust and S. Theusen, Lectures on String Theory (Springer, Berlin, 1989); J. Polchinski, String Theory (Cambridge University Press, Cambridge, England, 1998).

[7] B. Zwiebach, Phys. Lett. B 156, 315 (1985); B. Zumino, Phys. Rep. 137, 109 (1986).

[8] D. Lovelock, J. Math. Phys. 12, 498 (1971).

[9] N. D. Birrell and P. C. W. Davies, Quantum Fields in Curved Space (Cambridge University Press, Cambridge, England, 1982).

[10] I. Low and A. Zee, Nucl. Phys. B585, 395 (2000); J. M. Cline and H. Firouzjahi, Phys. Rev. D 65, 043501 (2002); P. Binetruy, C. Charmousis, S. C. Davis, and J. Dufaux, Phys. Lett. B 544, 183 (2002); C. Charmousis and J. Dufaux, Class. Quantum Grav. 19, 4671 (2002); S. Nojiri and S. D. Odintsov, J. High Energy Phys. 07, 049 (2000); J. E. Lidsey, S. Nojiri, and S. D. Odintsov, ibid. 06, 026 (2002); S. Nojiri, S. D. Odintsov and S. Ogushi, Int. J. Mod. Phys. A17, 4809 (2002); J. E. Lidsey and N. J. Nunes, Phys. Rev. D 67, 103510 (2003); I. P. Neupane, J. High Energy Phys. 09, 026 (2000); Y. M. Cho, I. P. Neupane and P. S. Wesson, Nucl. Phys B621, 388 (2002); Y. M. Cho and I. P. Neupane, Int. J. Mod. Phys. A18, 2703 (2003); M. A. Luty, M. Porrati, and R. Rattazi, J. High Energy Phys. 09, 029 (2003); J. P. Gregory and A. Padilla, Class. Quantum Grav. 20, 4221 (2003).

[11] D. G. Boulware and S. Deser, Phys. Rev. Lett. 55, 2656 (1985); R. G. Cai and K. S. Soh, Phys. Rev. D 59, 044013 (1999); R. G. Cai, ibid. 65, 084014 (2002); R. Aros, R. Troncoso, and J. Zanelli, ibid. 63, 084015 (2001).

[12] M. H. Dehghani, Phys. Rev. D 67, 064017 (2003); 69, 064024 (2004). 
[13] A. G. Riess et al., Astron. J. 116, 1009 (1998); S. Perlmutter et al., Astrophys. J. 517, 565 (1999); J. L. Tonry et al., Astrophys. J. 594, 1 (2003).

[14] A. T. Lee et al., Astrophys. J. 561, L1 (2001); C. B. Netterfield et al., Astrophys. J. 571, 604 (2002); N. W. Halverson et al. Astrophys. J. 568, 38 (2002); D. N. Spergel et al., Astrophys. J. Suppl. 148, 175 (2003).

[15] R. R. Caldwell, R. Dave and P. J. Steinhardt, Phys. Rev. Lett. 80, 1582 (1998); A. A. Starobinsky and V. Sahni, Int. J. Mod. Phys. D9, 373 (2000).

[16] S. Capozziello, Int. J. Mod. Phys. D11, 483 (2002); S. Capozziello, V. F. Cardone, S. Carloni, and A. Troisi, ibid. D12, 1969 (2003); S. Nojiri and S. D. Odintsov, Mod. Phys. Lett. A19, 627 (2004); hep-th/0308176.

[17] A. D. Dolgov and M. Kawasaki, Phys. Lett. B573, 1 (2003).

[18] C. Lanczos, Ann. Math. 39, 842 (1938).

[19] R. C. Myers, Phys. Rev. D 36, 392 (1987).

[20] S. C. Davis, Phys. Rev. D 67, 024030 (2003).

[21] M. Vissar, Class Quant. Grav. 21, 2603 (2004).

[22] A. G. Riess et al. Astrophys. J. 607, 665 (2004). 